\title{
Nanomedicine for vaccination and diagnosis of diseases
}

\author{
Stefan Lyer ${ }^{1}$, Christina Janko ${ }^{1}$, Ralf P Friedrich ${ }^{1}$, Iwona Cicha1 (i), Rainer Tietze ${ }^{1}$, Harald \\ Unterweger ${ }^{1}$ \& Christoph Alexiou*,1 \\ ${ }^{1}$ Department of Otorhinolaryngology, Head \& Neck Surgery, Section of Experimental Oncology \& Nanomedicine (SEON), Else \\ Kröner-Fresenius-Stiftung-Professorship, Universitätsklinikum Erlangen, Friedrich-Alexander-Universität Erlangen-Nürnberg, \\ Glueckstr. 10a, Erlangen 91054, Germany \\ *Author for correspondence: c.alexiou@web.de
}

First draft submitted: 28 December 2020; Accepted for publication: 18 January 2020; Published online: 3 February 2021

Keywords: cancer • disease detection • immunotherapy • vaccinations • viral infections

The severe acute respiratory syndrome coronavirus 2 (SARS-CoV-2) virus is currently the number one topic in media worldwide and is without question one of the biggest problems for the health of the global population. Nevertheless, it is and will not remain the only serious health problem that science and medicine, but also politics and society, have to face. Therefore, we have selected for this article some topics that deal with SARS-CoV-2 and other diseases that are thematically similar, but not related to the virus. The newest developments indicate that in particular, nanomedicine can, and will, play an important role in combating the current and future pandemics, as well as noninfectious diseases.

\section{Nanoparticulate mRNA vaccine against COVID-19}

Evaluation of: Zhang NN, Li XF, Deng YQ et al. A thermostable mRNA vaccine against COVID-19. Cell 182(5), 1271-1283.e16 (2020).

Zhang et al. describe the development of ARCoV, one of the mRNA-vaccines against the SARS-CoV-2-virus that is currently on its way to being or already approved for human use in some countries. The great advantage of mRNA is that nowadays it can be manufactured without the need of cell culture, removing the connected biosafety issues. In addition, new vaccines can be developed and produced very quickly by using mRNA, once the appropriate sequence is known [1]. The authors describe the synthesis of the lipid nanoparticles (LNP) that are used for encapsulation of the mRNA, which was modified from an existing protocol for small interfering RNAs, followed by a tangential flow filtration and purification. They used a fragment of the SARS-CoV-2 spike protein, the receptor-binding domain (RBD), as an immunization antigen. Transfecting this mRNA into four cell lines led to high production of the respective protein fragment, which was recognized by several antibodies and interacted with three positive patient sera. In Balb/c-mice, the injection of LNPs loaded with a luciferase non-Covid-mRNA-construct resulted in a photon flux in the muscle tissue around the injection site and in the liver. In muscle tissue, the LNPs triggered a massive infiltration of monocytes, macrophages and dendritic cells, acting as immune response adjuvants, as has been previously suggested [2]. Correspondingly, after injection of the final mRNA construct, RBD-staining was detected in these tissues that colocalized to macrophages in muscle, lymph nodes and liver tissue, showing that ARCoV-LNPs were able to recruit antigen-presenting cells. Subsequently, immunocompetent female BALB/c mice were immunized with single doses of 2 and $30 \mu \mathrm{g}$, or with doses of 2 and $10 \mu \mathrm{g}$, and a booster injection of the same doses at day 14. All injections induced production of SARS-CoV-2-specific IgGs and neutralizing antibodies with the double dosing of $10 \mu \mathrm{g} \mathrm{ARCoV}$ being the most effective regimen. Subsequently, the authors evaluated the in vivo protection efficacy of the vaccination 40 days after the second injection of 2 and $10 \mu \mathrm{g}$, respectively with 6000 plaque forming units of an adapted virus strain MASCp6. RNA measurement and immunostaining of the lungs and the upper respiratory tract showed full protection after vaccination and no clinical symptoms or tissue alterations, whereas both virus and clinical alterations were found in the placebo groups. As a second in vivo model, a placebo-controlled vaccination study with doses of 100 or $1000 \mu \mathrm{g}$ of ARCoV was performed 
in cynomolgus monkeys. Also in this model, efficient vaccination results were shown, albeit one of ten animals failed to produce detectable neutralizing antibodies and in comparison to other mRNA-vaccines the doses were relatively high [3]. Finally, the authors investigated the influence of different storage temperatures on the efficacy of the vaccine, demonstrating a good stability at room temperature within 7 days. This could be of high importance, when the vaccines have to be distributed to the population of underdeveloped regions far away from larger cities. Currently, the vaccine is being tested in a phase I placebo-controlled clinical study (ChiCTR2000034112).

- Written by S Lyer

\section{Cellular membrane nanodecoys for hijacking COVID-19 and inflammatory cytokines}

Evaluation of: Rao L, Xia S, Xu W et al. Decoy nanoparticles protect against COVID-19 by concurrently adsorbing viruses and inflammatory cytokines. Proc. Natl Acad. Sci. USA 117(44), 27141-27147 (2020).

SARS-CoV-2 has led to the ongoing global coronavirus disease 2019 (COVID-19) pandemic with over 92 million of cases and over 2 million deaths worldwide as of 16 January 2021 [4]. While vaccines are still under development, there is an urgent need for the effective therapeutic strategies. Coronaviruses contain positive-sense, single-stranded RNA and use the Spike protein (S Protein) for infection. To enter host target cells, the subunit S1 binds to the human receptor ACE2, and S2 facilitates viral fusion and entry [5]. Macrophages and monocytes respond to the pathogens by producing inflammatory cytokines, however, this can sometimes lead to cytokine release syndrome, with clinical symptoms such as septic shock and/or organ failure [6]. In their study, Rao et al. constructed decoy nanoparticles composed of fused cell membranes from human monocytes and genetically engineered cells stably expressing ACE2. Such particles, displaying a variety of cytokine receptors, are expected to compete with host cells for SARS-CoV-2 binding and neutralize cytokines. The nanodecoys were prepared by transducing ACE2 onto human embryonic kidney 293T cells. Afterward, cell membrane nanovesicles extracted from 293T/ACE2 and from human mononuclear THP-1 cells were fused by mixing, sonication and repeated extrusion through nanopores, to form $100 \mathrm{~nm}$-sized round lipid droplets. Expression of ACE2 for binding of SARS-CoV-2, CD130 for IL-6 and CD116 for GM-CSF was proven by western blotting. Next, the antiviral efficacy was tested in vitro by incubating SARS-CoV-2 pseudovirus with human hepatoma Huh-7 cells and nanodecoys. Pseudovirus infection of the Huh-7 cells was significantly reduced in the presence of ACE2 nanovesicles and nanodecoys, but not with 293T or THP1 vesicles. Furthermore, nanodecoys successfully hijacked the authentic SARS-CoV-2, dose-dependently reducing the viral copy numbers with negligible cytotoxicity. Nanodecoys also reduced IL-6 and GM-CSF levels in THP-1 cells stimulated with LPS. Finally, nanodecoys were administered in vivo via inhalation, showing excellent retention in the lungs of mice for $72 \mathrm{~h}$. Acute lung inflammation was provoked in mice by intratracheal inhalation of LPS, followed by nanodecoys inhalation $4 \mathrm{~h}$ later. After $8 \mathrm{~h}$, lung bronchalveolar lavage fluid was investigated, revealing reduced amounts of IL- 6 and GM-CSF, as well as lower total protein levels as compared with control animals. Moreover, nanodecoys dose-dependently suppressed inflammatory alveolar injuries and immune cell infiltration, as detected by histological examinations. In sum, nanodecoys effectively neutralized viruses and inflammatory cytokines, thus representing a promising antiviral nanotechnology with clear translational potential.

- Written by C Janko

\section{Immunotherapy of breast cancer by dendritic cell vaccines}

Evaluation of: Ni J, Song J, Wang B et al. Dendritic cell vaccine for the effective immunotherapy of breast cancer. Biomed Pharmacother. 126, 110046 (2020).

Immunotherapy has revolutionized the treatment of various types of cancer and the number of drugs approved for this indication is increasing. However, the controlled modulation of the immune system and the reduction of adverse side effects, such as nonspecific inflammation and autoimmunity, are still a major challenge. Additionally, the strong immunosuppressive impact of the breast cancer microenvironment has prevented successful treatment in the past. The use of T cells or dendritic cells (DCs) in combination with advanced drug delivery systems has the potential to improve cancer targeting and therapeutic efficiency [7]. DCs, being responsible for antigen processing and presentation and the activation of $\mathrm{T}$ cells in the initiation of the immune response, represent a particularly promising target for the development of a therapeutic vaccine [8].

A nanoparticle-based approach to the development a DC vaccine was recently described by $\mathrm{Ni}$ and colleagues [9]. They produced cancer cell membrane coated calcium carbonate nanoparticles (MC) containing tumor specific proteins. Doxorubicin hydrochloride (Dox) was encapsulated in the particle core to trigger cell death, whereas the photosensitizing chlorin e6 (Ce6) was loaded in the cell membrane to enable an effective photodynamic therapy 
due to the generation of reactive oxygen species (ROS). The nearly spherical MC/Dox/Ce6 particles showed a hydrodynamic size of approximately $100 \mathrm{~nm}$ and a zeta potential of $-16.14 \mathrm{mV}$. In contrast to very good stability and low Dox release at physiological $\mathrm{pH}$, the drug release was significantly higher at lower $\mathrm{pH}$, which is characteristic for tumor environment. In vitro experiments using the breast cancer cell line $4 \mathrm{~T} 1$ showed a strong inhibition of cell growth by these particles, which could be further enhanced by additional laser irradiation. Further experiments confirmed the laser-induced generation of elevated ROS amounts in presence of Ce6-containing particles. Using calreticulin antibodies, confocal microscopy and flow cytometry proved a strongly enhanced immunogenic cell death in presence of Dox, Ce6 and laser irradiation. In vivo experiments with $4 \mathrm{~T} 1$ tumor-bearing mice treated with MC/Dox/Ce6 particles and laser irradiation showed very high levels of IL-6, IL-12 and TNF- $\alpha$ in blood serum. Compared with the control groups, the elevated cytokine levels were still present after 4 days, indicating a successful vaccination and a continued activation of DC cells. Finally, the therapeutic potential of the immunotherapy was shown in mice carrying both primary and distant tumors. In contrast to the quickly evolving tumors in mice treated with PBS and Dox and the moderate growth of MC/Dox- and MC/Dox/Ce6-treated mice, tumors were significantly inhibited in mice after MC/Dox/Ce6 treatment and laser irradiation.

In summary, $\mathrm{Ni}$ and colleagues, presented a promising strategy for the generation of new cancer vaccines, combining chemotherapy, photodynamic therapy and immunotherapy.

- Written by RP Friedrich

\section{Detection of ultrasmall metastases using tumor-targeting nanoprobe-enhanced MRI}

Evaluation of: Li Y, Zhao X, Liu X et al. A bioinspired nanoprobe with multilevel responsive T1-weighted MR signal-amplification illuminates ultrasmall metastases. Adv. Mater. 32(4), 1906799 (2019).

Tumor metastases are the leading cause of death in cancer patients [10]. Magnetic resonance imaging (MRI) is an important tool for the non-invasive screening of tumors due to the high spatial resolution of this modality and $3 \mathrm{D}$ anatomical information [11]. However, the accurate MRI detection of ultrasmall metastases $(<1 \mathrm{~mm})$ which are composed of small clusters of cancer cells and lack the blood vessels, remains a challenge. An additional limiting factor in MRI detection of ultrasmall metastases is the low contrast agent accumulation, resulting in a weak signal at tumor sites.

To address this problem, Li et al. developed ultrasmall manganese ferrite nanoparticles (UMFNPs), which were conjugated with a tumor-targeting penta-peptide CREKA (Cys-Arg-Glu-Lys-Ala) to create an ultrasensitive T1weighted magnetic resonance imaging (MRI) contrast agent (UMFNP-CREKA). The size of UMFNP-CREKA in aqueous solution was $5.7 \mathrm{~nm}$ and their zeta-potential was $-12.4 \mathrm{mV}$. Doping with manganese increased the $\mathrm{r} 1$ relaxivity of UMFNP-CREKA to $6.79 \mathrm{~mm}^{-1} \mathrm{~s}^{-1}$, which was about $\approx 1.8$-times higher than that of $\gamma-\mathrm{Fe}_{2} \mathrm{O}_{3}$ CREKA. In response to the unique neoplastic microenvironment of metastases, characterized by the high amounts and concentration-gradient of $\mathrm{H}^{+}$and $\mathrm{H}_{2} \mathrm{O}_{2}$, UMFNPs exhibited the ability to catalyze $\mathrm{H}_{2} \mathrm{O}_{2}$. Furthermore, the release of $\mathrm{Mn}^{2+}$, which is known to amplify MR signals by interacting with surrounding proteins [12], was strongly accelerated at the low $\mathrm{pH}$ and in the presence of $\mathrm{H}_{2} \mathrm{O}_{2}$.

Due to a high binding affinity with fibronectin, CREKA is expected to target the metastatic tumor edge. The authors showed an efficient accumulation of UMFNP-CREKA-Cy5.5 and CREKA-Cy5.5 compared with free Cy5.5 in the tumor sites of mice bearing subcutaneous 4T1-Luc breast tumors. Subsequently, the mice were administered UMFNP-CREKA $\left(5.0 \mathrm{mg} \mathrm{kg}^{-1}[\mathrm{Fe}+\mathrm{Mn}]\right)$, followed by T1-weighted MRI measurements. Compared with control particles $\left(\gamma-\mathrm{Fe}_{2} \mathrm{O}_{3}\right.$-CREKA or UMFNP-DHCA), UMFNP-CREKA exhibited the strongest signal enhancement and highest CNR in both the tumor periphery and interior for the 120 min post injection.

Because breast cancer has a high rate of lung metastases [13], the authors established a lung metastasis model by tail vein inoculation of mice with 4T1-Luc breast cancer cells. At day 10 post inoculation, the UMFNP-CREKAenhanced MR images revealed multiple spots in the lung, and a metastasis as small as $0.39 \mathrm{~mm}$ in diameter was successfully detected. No detectable metastatic breast cancer contrast enhancement was observed in the lung in the T1-weighted MR images in the $\gamma$ - $\mathrm{Fe}_{2} \mathrm{O}_{3}$-CREKA and UMFNP-DHCA groups. In the spontaneous metastatic breast tumor model developed by the authors, the metastases spread throughout the body (including the lung, bone, lymph nodes and adrenal glands) were effectively detected by UMFNP-CREKA at 30 min post injection.

In conclusion, the bioinspired nanoprobe reported by Li et al. represents an efficient strategy for the detection of ultrasmall breast cancer metastases, significantly extending the detection limit of the current imaging modalities.

- Written by I Cicha 


\section{Versatile and multivalent nanobodies efficiently neutralize SARS-CoV-2}

Evaluation of: Xiang Y, Nambulli S, Xiao Z et al. Versatile and multivalent nanobodies efficiently neutralize SARS-CoV-2. Science 370(6523), 1479-1484 (2020).

The still critical situation regarding COVID-19 pandemic requires extensive research efforts in the field of diagnostics and therapy. There is a readiness to take unconventional approaches in order to develop effective therapies, especially for severe courses of the disease. A promising concept is based on the application of socalled nanobodies as neutralizing antibodies, which effectively prevent the viruses from binding to the host cells. Nanobodies are generated from single strain of antibodies produced in Camelidae species in immune response to various pathogens [14].

In the study by Xiang et al., llamas were immunized with a recombinant protein representing the RBD of SARS-CoV-2. The animals showed a potent and specific immune response in the form of a high antibody titer of $1.75 \times 10^{6}$. The obtained serum had the ability to effectively neutralize pseudotyped SARS-CoV-2, with halfmaximal neutralization titer (NT50) of $\sim 310,000$, which was orders of magnitude more effective than serum from human convalescents that has maximum neutralization levels around 5,000 [15]. For further characterization, single chain $\mathrm{V}_{\mathrm{H}} \mathrm{H}$ antibodies were isolated from this serum, which were shown to have high binding affinities against the $\mathrm{RBD}$ of the virus $\left(\mathrm{IC}_{50}=509 \mathrm{pM}\right)$. In an extensive proteomic analysis, 109 highly diverse nanobody sequences were selected from thousands of different $\mathrm{V}_{\mathrm{H}} \mathrm{H}$ antibodies and submitted to E. coli expression. The resulting recombinant proteins were further investigated in binding studies using ELISA and SARS-CoV-2-GFP pseudovirus neutralization assays. Among them, $6 \%$ had neutralization activities below $0.5 \mathrm{nM}$. Subsequently, the ability of these candidates to neutralize the SARS-CoV-2 Munich strain was examined. All candidates achieved full neutralization in a concentration-dependent manner. The three best nanobodies had values of $2.1 \mathrm{ng} / \mathrm{ml}(0.133 \mathrm{nM}), 1.6 \mathrm{ng} / \mathrm{ml}$ $(0.102 \mathrm{nM})$ and $0.7 \mathrm{ng} / \mathrm{ml}(0.045 \mathrm{nM})$, with a stable binding to the target structure. The affine candidate was also tested for on-shelf stability and remained soluble after $\sim 6$ weeks of storage at room temperature after purification. No multimeric forms or aggregations were detected by size-exclusion chromatography. It can thus be concluded that the described nanobodies exhibit excellent physicochemical properties and could serve as effective therapeutic tool in the context of SARS-CoV-2 infections. These nanobodies represent the most potent SARS-CoV-2 neutralizers to date. Future developments should focus on the application technique to allow safe administration in the most effective form.

- Written by R Tietze and C Alexiou

\section{Effective cancer immunotherapy based on injectable DNA vaccine delivery scaffolds}

Evaluation of: Nguyen TL, Yin Y, Choi Y, Jeong JH, Kim J. Enhanced cancer DNA vaccine via direct transfection to host DCs recruited in injectable scaffolds. ACS Nano 14(9), 11623-11636 (2020).

Globally, one in six deaths is due to cancer, making it one of the most deadly diseases of our time [16]. This challenging condition is approached from different perspectives, one of them being the improvement of the therapy outcome. In this context, developments in the field of cancer immunotherapy are particularly promising.

In their work, Nguyen et al. developed an enhanced cancer DNA vaccine based on polyplex and injectable scaffolds, which act as a host for direct transfection of recruited DCs. Polyplex consists of plasmid DNA encoding ovalbumin, a model antigen protein used in cancer vaccine studies, and branched PEI. The basis of the scaffolds are high-aspect-ratio mesoporous silica microrods (MSR) with an average length of $80 \mu \mathrm{m}$, a width of $14.5 \mu \mathrm{m}$, and a pore volume of $1.04 \mathrm{~cm}^{3} / \mathrm{g}$. These MSRs are loaded with the DC-recruiting chemokine GM-CSF and with $\mathrm{PEI}$, as a vaccine potentiator to enhance the immunogenicity of the DNA vaccine $\mathrm{CpG}$ oligodeoxynucleotides $(\mathrm{CpG})$ which are also incorporated in the MSRs. CpG is a potent immune adjuvant and has been widely used in clinical trials. However, dedicated facilities and special training is required for the application, which furthermore often resulted in discomfort and pain to the patients [17]. These drawbacks can be overcome by the approach of the proposed MSR-based system, which can be injected into the subcutaneous tissue.

The potential of this system to directly deliver DNA polyplexes into DCs through subcutaneous injection of the DC-recruiting MSR scaffolds was successfully demonstrated in mice. To test the system in a prophylactic setup, B16-OVA cells were used to induce tumor growth in mice. Compared with the untreated control animals, in mice immunized with the DNA vaccine ten days before the B16-OVA inoculation, the tumor growth was significantly suppressed, most likely by the induction of a robust antigen-specific adaptive immune response. In a mouse lung cancer model, the DNA vaccine was capable of significantly suppressing the occurrence of metastasis. 
It is important to note that the combination of the DNA vaccine with anti-PD1, an anti-programmed cell death-1 antibody, resulted in an almost normal histopathology of the lungs.

Taken together, Nguyen et al. provided extensive proof-of-concept studies to show the remarkable performance of their MSR-based DNA vaccine for improved cancer immunotherapy.

- Written by $\mathrm{H}$ Unterweger

\section{Financial \& competing interests disclosure}

The authors have no relevant affiliations or financial involvement with any organization or entity with a financial interest in or financial conflict with the subject matter or materials discussed in the manuscript. This includes employment, consultancies, honoraria, stock ownership or options, expert testimony, grants or patents received or pending, or royalties.

No writing assistance was utilized in the production of this manuscript.

\section{References}

1 Tombacz I, Weissman D, Pardi N. Vaccination with messenger RNA: a promising alternative to DNA vaccination. In: DNA Vaccines. Methods in Molecular Biology (Volume 2197). Sousa Â (Ed.). Humana, NY, USA, 13-31 (2021).

2 Maugeri M, Nawaz M, Papadimitriou A et al. Linkage between endosomal escape of LNP-mRNA and loading into EVs for transport to other cells. Nat. Commun. 10, 4333 (2019).

3 Walsh EE, Frenck RW Jr, Falsey AR et al. Safety and immunogenicity of two RNA-based Covid-19 vaccine candidates. N. Engl. J. Med. 383(25), 2439-2450 (2020).

4 World Health Organization. WHO Coronavirus Disease (COVID-19) Dashboard. https://covid19.who.int/

5 Hoffmann M, Kleine-Weber H, Schroeder S et al. SARS-CoV-2 cell entry depends on ACE2 and TMPRSS2 and is blocked by a clinically proven protease inhibitor. Cell 181(2), 271-280 (2020).

6 Huang C, Wang Y, Li X et al. Clinical features of patients infected with 2019 novel coronavirus in Wuhan, China. Lancet 395(10223), 497-506 (2020).

7 Riley RS, June CH, Langer R, Mitchell MJ. Delivery technologies for cancer immunotherapy. Nat. Rev. Drug. Discov. 18(3), 175-196 (2019).

8 Santos PM, Butterfield LH. Dendritic cell-based cancer vaccines. J. Immunol. 200(2), 443-449 (2018).

9 Ni J, Song J, Wang B et al. Dendritic cell vaccine for the effective immunotherapy of breast cancer. Biomed. Pharmacother. 126, 110046 (2020).

10 Cedolini C, Bertozzi S, Londero AP et al. Type of breast cancer diagnosis, screening, and survival. Clin. Breast Cancer 14(4), 235-240 (2014).

11 Van Zelst JCM, Vreemann S, Witt HJ et al. Multireader study on the diagnostic accuracy of ultrafast breast magnetic resonance imaging for breast cancer screening. Invest. Radiol. 53(10), 579-586 (2018).

12 Lelyveld VS, Brustad E, Arnold FH, Jasanoff A. Metal-substituted protein MRI contrast agents engineered for enhanced relaxivity and ligand sensitivity. J. Am. Chem. Soc. 133(4), 649-651 (2011).

13 Lee M, Heo SH, Song IH et al. Presence of tertiary lymphoid structures determines the level of tumor-infiltrating lymphocytes in primary breast cancer and metastasis. Mod. Pathol. 32(1), 70-80 (2019).

14 Zare H, Rajabibazl M, Rasooli I et al. Production of nanobodies against prostate-specific membrane antigen (PSMA) recognizing LnCaP cells. Int. J. Biol. Marker. 29(2), E169-E179 (2014).

15 Robbiani DF, Gaebler C, Muecksch F et al. Convergent antibody responses to SARS-CoV-2 in convalescent individuals. Nature 584(7821), 437-442 (2020).

16 World Health Organization. WHO Report on Cancer: Setting Priorities, Investing Wisely and Providing Care for All. World Health Organization, Geneva, Switzerland (2020).

17 Vollmer J, Krieg AM. Immunotherapeutic applications of CpG oligodeoxynucleotide TLR9 agonists. Adv. Drug. Deliv. Rev. 61(3), 195-204 (2009). 
